\title{
Interaction symbolique dans le non-lieu liminal : une lecture microsociologique de Bernard-Marie Koltès
}

\author{
Ignasi RiBó \\ Mae Fah Luang University \\ ignasi.rib@mfu.ac.th \\ https://orcid.org/0000-0001-7821-316x
}

\section{Resumen}

Este ensayo interpreta la obra de Bernard-Marie Koltès Dans la solitude des champs de coton (1986) desde el punto de vista de la microsociología de Erving Goffman. La interacción simbólica entre el Cliente y el Vendedor es analizada como una forma de ritual performativo desplegado en el no-lugar liminal de la supermodernidad, donde dos desconocidos se enzarzan en un concurso de caracteres con consecuencias fatales. Dado que ninguno de los dos es capaz de retirarse del enfrentamiento sin sufrir un insulto a la sacralidad del rostro, no pueden alcanzar un compromiso utilitario. La lucha, llevada a cabo de manera altamente ritualizada, comporta el fracaso del sistema social emergente.

Palabras clave: drama, ritual, conflicto, posthumanismo, semiótica.

\section{Résumé}

Cet essai interprète la pièce de Bernard-Marie Koltès Dans la solitude des champs de coton (1986) du point de vue de la microsociologie d'Erving Goffman. L'interaction symbolique entre le Client et le Dealer est analysée comme une forme de rituel performatif déployé dans le non-lieu liminal de la surmodernité, où deux étrangers se livrent à un concours de caractères aux conséquences fatales. Puisqu'aucun d'eux n'est capable de se retirer de la confrontation sans subir une offense au sacré du visage, un compromis utilitariste ne peut être atteint. La lutte, menée de manière hautement ritualisée, comporte l'échec du système social émergent.

Mots clé : drame, rituel, conflit, posthumanisme, sémiotique.

\begin{abstract}
This essay interprets Bernard-Marie Koltès' play Dans la solitude des champs de coton (1986) from the point of view of Erving Goffman's microsociology. The symbolic interaction between the Client and the Dealer is analysed as a form of performative ritual deployed in the liminal non-place of supermodernity, where two strangers engage in a character contest with fatal consequences. Since neither of them can withdraw from the confrontation without
\end{abstract}

*Artículo recibido el 17/12/2020, aceptado el 14/03/2021. 
suffering a slur to the sacredness of the face, a utilitarian compromise cannot be reached. The struggle, carried out in a highly ritualised manner, entails the failure of the emerging social system.

Keywords: drama, ritual, conflict, posthumanism, semiotics.

\section{Introduction}

Le but de cet essai est de donner une interprétation de la pièce Dans la solitude des champs de coton (1986) de Bernard-Marie Koltès en utilisant quelques concepts empruntés de la microsociologie d'Erving Goffman. Développé dans les années 1950 et 1960, le travail sociologique de Goffman visait surtout à décrire et analyser les interactions les plus quotidiennes entre les individus, conversations, rencontres, civilités ou rassemblements, en tant qu'elles produisent une certaine forme d'ordre social qu'il désignait comme " ordre de l'interaction" (Cefaï \& Perreau, 2012).

L'étude d'un texte dramatique avec des concepts dérivés, au moins en partie, des traditions et des pratiques théâtrales nous oblige à prendre certaines précautions méthodologiques. Il est bien connu que le travail de Goffman repose dans une large mesure sur l'application d'un modèle dramaturgique pour interpréter et expliquer les interactions sociales. Goffman (1959 : 254) a souligné les limites de cette analogie : "An action staged in a theater is a relatively contrived illusion and an admitted one; unlike ordinary life, nothing real or actual can happen to the performed characters". Ceci a été ajouté à la deuxième édition de The Presentation of Self in Everyday Life en tant que Goffman ressentait le besoin de justifier son utilisation du vocabulaire et des concepts dramaturgiques, tout en distinguant son travail sociologique de l'analyse de la performance théâtrale.

Cet essai se propose, cependant, d'appliquer ces mêmes concepts à l'analyse d'une pièce de théâtre, en inversant en quelque sorte le chemin tracé par Goffman luimême. Cette approche, inspirée des idées poststructuralistes développées dans le sillage des travaux de Goffman, notamment l'anthropologie de Marc Augé, la philosophie politique de Giorgio Agamben et la pensée posthumaniste en général, repose sur une ontologie plus fluide et relationnelle, où les frontières entre pratiques discursives (sémiotiques) et sociales (matérielles) sont fondamentalement bouleversées (Ribó, 2020a ; 2020b). Partant de ces prémisses, j'examinerai deux aspects de la conversation entre le Client et le Dealer dans la pièce de Koltès : le lieu de l'action et les rituels d'interaction. Les deux aspects sont, évidemment, étroitement liés. Si je les ai traités séparément, c'est exclusivement pour des raisons de clarté méthodologique. Ainsi, j'espère décrire, en utilisant le langage de la microsociologie, une rencontre qui n'a jamais eu lieu, mais qui, en quelque sorte, a lieu tous les jours. 


\section{Le lieu de l'action}

"Un deal », nous prévient l'auteur d'emblée, " est une transaction commerciale [...] qui se conclut, dans des espaces neutres, indéfinis, et non prévus à cet usage " (Koltès, 1986 : 7). Et pourtant, l'espace de cette transaction, l'espace où se rencontrent le Dealer et le Client, a certaines caractéristiques bien déterminées : c'est urbain, nocturne et clandestin. Si l'on tient compte des éléments descriptifs que les deux protagonistes apportent au cours du dialogue, on peut facilement imaginer une ruelle marginale à Marseille, Los Angeles, Manille ou toute autre métropole contemporaine. Cela seul ne permet cependant pas de situer les actions des personnages ou de comprendre le type d'interaction sociale qui s'y produit. En fait, nous ne pouvons spécifier que quelques éléments de l'" espace " physique, dans la terminologie de Marc Augé (1995: 73), alors que c'est le « lieu anthropologique » qui nous intéresse vraiment. Pour interpréter cette interaction, nous devons commencer donc par comprendre plus précisément l'endroit où la rencontre entre le Client et le Dealer a lieu. En fait, qu'est-ce que le champ de coton koltésien?

Selon Augé (1992 : 73), un lieu anthropologique «n'est que l'idée, partiellement matérialisée, que se font ceux qui l'habitent de leur rapport au territoire, à leurs proches et aux autres ». Ce genre de lieu, agencé par des multiples formes géométriques, notamment par des itinéraires, carrefours et centres, est un espace intensif en identité, en relations et en histoire. C'est donc un espace d'appartenance, où se produisent des formes traditionnelles de socialisation, des réseaux riches et des enchevêtrements organiques entre les acteurs.

Dans le champ de coton koltésien, on peut certainement trouver les formes géométriques qui, selon Augé, caractérisent le lieu anthropologique. Mais ici elles ont des fonctions assez différentes. Ainsi, dans le champ de coton la ligne n'est plus un itinéraire mais une trajectoire (Koltès, $1986: 13$ ) :

Je ne marche pas en un certain endroit et à une certaine heure ; je marche, tout court, allant d'un point à un autre, pour affaires privées qui se traitent en ces points et non pas en parcours; je ne connais aucun crépuscule ni aucune sorte de désirs et je veux ignorer les accidents de mon parcours.

De même, l'intersection n'est plus un carrefour mais une collision (Koltès, $1986: 17)$ :

Car, quoi que vous en disiez, la ligne sur laquelle vous marchiez, de droite peut-être qu'elle était, est devenue tordue lorsque vous m'avez aperçu, et j'ai saisi le moment précis où vous m'avez aperçu par le moment précis où votre chemin devint courbe, et non pas courbe pour vous éloigner de moi, mais courbe pour venir à moi, sinon nous ne nous serions jamais rencontrés. 
Et enfin, le point d'intersection n'est plus un centre mais un labyrinthe (Koltès, 1986 : 20) : "la ligne droite, censée me mener d'un point lumineux à un autre point lumineux, à cause de vous devient crochue et labyrinthe obscur dans l'obscur territoire où je me suis perdu».

Ce que nous avons donc ici, c’est ce qu'Augé (1992 : 119) appelle un « nonlieu ", c'est-à-dire un espace où manquent l'identité, les relations et l'histoire, un espace où la solidarité organique cède la place à la « contractualité solitaire ». D'une certaine façon, le champ de coton peut être vu comme une représentation dramatique des espaces archétypiques de la postmodernité (ou la "surmodernité ", selon la terminologie d'Augé) : aéroports, autoroutes, supermarchés, ainsi que les rues urbaines et les ruelles sombres de la vie nocturne et du commerce illégal.

Ceci n'est pas sans conséquences. Le non-lieu qu'est le champ de coton détermine l'interaction à son niveau le plus fondamental. Comme le fait remarquer Augé (1992: 128-129) :

[...] l'utilisateur du non-lieu est toujours tenu de prouver son innocence. [...] Mais l'innocence, c'est encore autre chose : l'espace du non-lieu délivre celui qui y pénètre de ses déterminations habituelles. Il n'est plus que ce qu'il fait ou ce qu'il vit comme passager, client, conducteur.

Dans ce non-lieu qu'est le champ de coton, le Dealer n'est que quelqu'un qui cherche à vendre et le Client n'est que quelqu'un qui cherche à acheter. Toute autre identité ou subjectivité, qui dans un autre espace ou à un autre moment aurait pu être importante, devient ici sans objet. Et c'est en ce sens que le Client et le Dealer se trouvent juste un pas au-dessus ou au-delà de ce que Giorgio Agamben (1997) appelle la "vie nue " (zoe), le statut non-qualifié des corps vivants en dehors des frontières de la communauté politique. Sans nom, sans pays, sans famille, sans amis, sans aucun autre attachement social, tous les deux risquent tout dans cette rencontre.

Le concept négatif du non-lieu peut nous aider à mieux comprendre la nature de l'espace dans lequel se déploie cette interaction symbolique entre le Dealer et le Client. Mais il nous faut compléter sa description en nous tournant vers un autre dispositif conceptuel. Dans son travail anthropologique, Victor Turner (1969: 96) a distingué

[...] two major " models» for human interrelatedness, juxtaposed and alternating. The first is of society as a structured, differentiated, and often hierarchical system of politico-legal-economic positions [...] The second, which emerges recognizably in the liminal period, is of society as an unstructured or rudimentarily structured and relatively undifferentiated comitatus, community, or even communion of equal individuals who submit together to the general authority of the ritual elders. 
Avec quelques mises en garde, nous pourrions dire que le Client est un individu qui vient du premier système et entre dans le second : "Mon commerce à moi ", affirme-t-il, " je le fais aux heures homologuées du jour, dans les lieux de commerce homologués et illuminés d'éclairage électrique "(Koltès, 1986 : 18). Ainsi, la descente du Client, provenant des étages supérieurs et s'aventurant dans ce champ de coton sombre et menaçant, retrace son entrée dans un monde marginal, une transition à travers le seuil ou limen qui s'étend entre deux étapes du monde stable au-dessus. "J'allais de cette fenêtre éclairée, derrière moi, là-haut, à cette autre fenêtre éclairée, là-bas devant moi, selon une ligne bien droite qui passe à travers vous parce que vous vous y êtes délibérément placé » (Koltès, 1986 : 13). En pénétrant dans le champ de coton, le Client devient donc un personnage liminal : " je suis ici, en parcours, en attente, en suspension, en déplacement, hors-jeu, hors vie, provisoire, pratiquement absent » (Koltès, $1986: 19)$.

Suivant ce raisonnement, on pourrait conclure que l'interaction entre le Client et le Dealer est un rite de passage, où le Dealer serait la figure marginale qui habite le limen et met en place le rituel d'initiation, tandis que le Client serait le néophyte qui s'y soumet. Cette interprétation est certes possible, mais soulève beaucoup de questions, surtout lorsqu'on l'examine dans le cadre de la théorie de Turner. Si nous regardions l'interaction comme un rite de passage, il faudrait accepter que le champ de coton soit une communitas. Mais cette forme de socialité implique, selon Turner, des liens organiques particulièrement forts entre les initiés. En revanche, comme nous le verrons tout de suite, l'interaction du Dealer et du Client est conflictuelle, très loin du communautarisme liminal décrit par Turner. De même, il est difficile d'accepter que le Dealer puisse représenter en quelque façon l'aîné qui contrôle le rituel, puisque le rapport entre le Dealer et le Client n'est pas hiérarchique, mais purement contractuel.

En effet, si le champ de coton est bien un limen, de même que le Client et le Dealer constituent des personnes liminaires, le passage auquel le premier est confronté ne correspond pas au modèle ethnologique de Turner, précisément parce qu'il a lieu dans un non-lieu. Les transitions rituelles dans des mondes riches de sens, comme les mondes des Tallensis ou des Nuer décrits par les ethnologues, mais aussi dans les sociétés dites modernes, peuvent certainement se produire dans des lieux liminaux, en communitas. Les transitions dans la surmodernité, en revanche, ne pourraient se produire que dans des non-lieux liminaux, dans des espaces qui, comme le champ de coton, combinent la détermination négative du non-lieu (manque d'identité, de relations et d'histoire) avec l'extrême ritualisation des interactions qui caractérise le seuil ou $l i$ men.

C'est justement cette ritualisation liminale qui nous permet de différencier le champ de coton des non-lieux analysés par Augé, comme les autoroutes, les aéroports ou les salles d'attente. Même si tous ces endroits peuvent être conçus comme des espaces de la surmodernité, les interactions dans ces espaces se déroulent en général dans 
les limites de l'expérience commune. Ce qui se passe là-dedans, ce sont des actions qui tendent à être, comme dit Goffman, problématiques sans avoir de conséquences ou avoir des conséquences sans être problématiques. Dans le champ de coton, au contraire, on assiste à une interaction extraordinaire où les acteurs s'engagent et sont confrontés à des actions à la fois conséquentes et problématiques, c'est-à-dire à des actions fatidiques (Goffman, 1967 : 171) :

There are extraordinary niches in social life where activity is so markedly problematic and consequential that the participant is likely to orient himself to fatefulness prospectively, perceiving in these terms what it is that is taking place. It is then that fateful situations undergo a subtle transformation, cognitively reorganized by the person who must suffer them. [...] Fateful situations become chancy undertakings, and exposure to uncertainty is construed as willfully taking a practical gamble.

La fatalité de la situation est précisément ce qui caractérise le non-lieu liminal où le Client et le Dealer se rencontrent. Dans cette situation, réduits à leur seule capacité d'acheter ou de vendre, qui est la seule qualification qui les élève au-dessus de leur vie nue, tous deux sont contraints d'agir. Et ici, le verbe agir ne veut pas dire simplement se comporter, mais fait référence à des activités qui sont conséquentes, problématiques et entreprises pour ce qui est ressenti comme étant leur propre raison (Goffman, 1967 : 185). Si les actions du Client et du Dealer dans le champ de coton sont tellement problématiques et conséquentes, c'est parce que tous les deux ont besoin de gagner ; tous les deux sont contraints de tout risquer pour sortir victorieux de cette rencontre fatidique ; tous les deux sont emmêlés dans une sorte d'interaction très particulière, un jeu fatal dans lequel le seul moyen de ne pas perdre est de s'imposer sur l'autre.

Ainsi, le conflit entre le Dealer et le Client prend la forme de ce que Goffman (1967 : 240) appelle un « concours de caractères » :

During occasions of this kind of action, not only will character be at stake, mutual fatefulness will prevail in this regard. Each person will be at least incidentally concerned with establishing evidence of strong character, and conditions will be such as to allow this only at the expense of the character of the other participants. The very field that the one uses to express character may be the other's character expression. And at times the primary properties at play may themselves be openly made a convenience, pointedly serving merely as an occasion for doing batthe by and for character. A character contest results; a special kind of moral game.

Le champ de coton est donc le non-lieu d'une confrontation incontournable, d'un concours de caractères comparable, dans l'espace de la surmodernité, à l'agôn tragique. Le Dealer et le Client s'y font face, tous deux contraints de gagner, chacun forcé 
de vaincre l'autre pour endurer cette rencontre fatidique et franchir le seuil ou limen (Koltès, $1986: 18)$ :

En toute fin de compte n'existe que le fait que vous m'avez regardé et que j'ai intercepté ce regard ou l'inverse, et que, partant, d'absolue qu'elle était, la ligne sur laquelle vous vous déplaciez est devenue relative et complexe, ni droite ni courbe, mais fatale.

\section{Les rituels d'interaction}

Comme il arrive dans les vrais duels, les rituels déployés dans cette situation sont voués à être particulièrement intenses. Afin de mieux comprendre cette dangereuse cérémonie entre le Dealer et le Client, il est nécessaire de déterminer en premier lieu l'objet réel de la confrontation. Apparemment, il s'agit d'une transaction commerciale assez banale, un échange de marchandises indéterminées entre un acheteur et un vendeur: "Si vous marchez dehors, à cette heure et en ce lieu, c'est que vous désirez quelque chose que vous n'avez pas, et cette chose, moi, je peux vous la fournir " (Koltès, 1986 : 9). Mais il ne faut pas oublier que tout ceci se passe dans le limen, à l'heure des " rapports sauvages entre les hommes et les animaux" (Koltès, $1986: 9$ ), pas "aux heures homologuées du jour " (Koltès, 1986 : 18). Il ne s'agit pas d'un simple échange commercial, mais d'une lutte pour l'hégémonie. La confrontation n'est cependant pas tant physique que symbolique. S'engageant dans cette interaction dialogique, soutenue par la menace implicite mais toujours présente de la violence, le Dealer et le Client essaient de défendre leur caractère en s'imposant sur l'autre. Comme dit Goffman (1967 : 241-242),

$[\ldots]$ this dispute will embarrass not only the desire for a satisfactory place in the definitions that prevail, but also the right to be given such a place and the duty to insist thereon. $\mathrm{A}$ " matter of principle " is involved, that is, a rule whose sanctity derives not only from the actual conduct that is guided by it, but also from its symbolic implication as one of a whole set of rules, the system itself being in jeopardy.

C'est pourquoi la transaction elle-même n'est en réalité pas aussi importante que d'imposer sa volonté à l'autre, en se portant mieux que son homologue, pour que l'on puisse repartir comme le gagnant d'une rencontre qui a moins à voir avec le commerce qu'avec la survie. C'est un enjeu vite reconnu par le Client lui-même (Koltès, $1986: 24)$ :

Il n'y a point de paix ni de droit dans les éléments naturels, il n'y a pas de commerce dans le commerce illicite, il n'y a que la menace et la fuite et le coup sans objet à vendre et sans objet à acheter et sans monnaie valable et sans échelle des prix, ténèbres, ténèbres des hommes qui s'abordent dans la nuit. 
Cela ne veut pas dire, cependant, que l'interaction entre le Dealer et le Client soit simplement " un rapport sauvage " (Koltès, $1986: 22$ ). Au contraire, l'absence de règles et de lois qui caractérise le non-lieu liminal exige une ritualisation extrême des échanges, qu'ils soient matériels ou sémiotiques, afin de préserver l'ordre cérémonial sans lequel la confrontation des caractères n'aurait aucun effet symbolique, de la même manière que la pratique de guérison du shaman est impuissante en dehors du rituel dont il puise toute son "efficacité symbolique " (Lévi-Strauss, 1974 : 205-226). C’est ainsi que le Dealer avertit le Client (Koltès, 1986 : 22) :

L'heure du crépuscule à laquelle vous vous êtes approché de moi est celle où la correction n'est plus obligatoire et devient donc nécessaire, où plus rien n'est obligatoire qu'un rapport sauvage dans l'obscurité, et j'aurais pu tomber sur vous comme un chiffon sur la flamme d'une bougie, j'aurais pu vous prendre par le col de la chemise, par surprise. Et cette correction, nécessaire mais gratuite, que je vous ai offerte, vous lie à moi.

Cette attitude du Dealer montre, comme dit Goffman (1967 : 247), «the cooperativeness and regard for rules that are required on the part of all participants if the game is to be successful in generating and jeopardizing character ». On assiste donc à une interaction hautement ritualisée, une cérémonie où la dynamique apparemment contradictoire de coopération et de confrontation doit être en quelque sorte intégrée, et où l'accord mutuel, la transaction, ne deviendra possible que si l'un des deux participants accepte son infériorité.

Comme le souligne Georg Simmel (1997 : 112), le rituel social commence souvent par le regard, qui permet à un étranger d'en interpeller un autre au milieu du flux urbain. L'interpellation n'est que le début de l'interaction, une offre aussi bien qu'une demande d'attention qui peut être vécue comme une opportunité mais aussi comme une menace. Dans le non-lieu liminal, l'interpellation est l'action qui transforme une trajectoire en une collision, le premier mouvement du concours de caractères, la provocation initiale. C'est pourquoi il est si important de déterminer qui a été le premier à regarder, qui a eu l'initiative, le Dealer ou le Client. Si l'interaction a été initiée par le Client, cela signifie qu'il cherche à satisfaire un désir au milieu de la nuit et se trouve donc en position de faiblesse par rapport au Dealer. Pour cette raison, le Client essaie de prouver qu'il a été interpellé par le Dealer et non à l'inverse, dans l'espoir de montrer que c'est le Dealer qui cherche à vendre au milieu de la nuit, ce qui lui permettrait de préserver la force de refuser toute marchandise que celui-ci pourrait lui offrir. Loin d'être un geste neutre et insignifiant, le regard devient donc une arme pointue aux conséquences fatales (Koltès, $1986: 22-23$ ) :

Si toutefois je l'ai fait, sachez que j'aurais désiré ne pas vous avoir regardé. Le regard se promène et se pose et croit être en terrain neutre et libre, comme une abeille dans un champ de fleurs, 
comme le museau d'une vache dans l'espace clôturé d'une prairie. Mais que faire de son regard?

Dans un non-lieu structuré comme l'aéroport ou le supermarché, le regard dirigé vers un inconnu prend la forme anodine de ce que Goffman (1963: 84) appelle " inattention civile », une reconnaissance polie de la présence mais aussi du manque de pertinence de l'étranger :

What seems to be involved [dans l'inattention civile] is that one gives to another enough visual notice to demonstrate that one appreciates that the other is present (and that one admits openly to having seen him), while at the next moment withdrawing one's attention from him so as to express that he does not constitute a target of special curiosity or design.

Dans le champ de coton, en revanche, le regard devient un défi, le premier coup dans un concours de caractères. Et, comme tout acte d'agression, il exige une résolution, ou pour le moins une riposte (Koltès, 1986 : 31) :

La vraie seule cruauté [dit le Dealer] de cette heure du crépuscule où nous nous tenons tous les deux n'est pas qu'un homme blesse l'autre, ou le mutile, ou le torture, ou lui arrache les membres et la tête, ou même le fasse pleurer ; la vraie et terrible cruauté est celle de l'homme ou de l'animal qui rend l'homme ou l'animal inachevé, qui l'interrompt comme des points de suspension au milieu d'une phrase, qui se détourne de lui après l'avoir regardé, qui fait, de l'animal ou de l'homme, une erreur du regard.

Une fois que la collision s'est produite, l'interaction entre le Dealer et le Client est marquée par le besoin, partagé par tous les deux, d'avoir le dessus, de vaincre l'autre dans le concours de caractères engagé, tout en préservant en même temps l'équilibre rituel. Toute la dynamique de l'échange dialogique découle donc de l'injonction contradictoire que Gregory Bateson (1980:11) a appelé "double contrainte " [double bind] : " quoi que fasse un individu pris dans cette situation "il ne peut pas être gagnant" ". Ainsi, le Dealer et le Client sont obligés de s'imposer l'un sur l'autre, mais ce faisant, ils briseraient l'enchevêtrement cérémoniel et réduiraient leur rencontre à un "rapport sauvage " (Koltès, 1986 : 22), un chaos indifférencié dans lequel le concours de caractères ne pourrait être plus résolu et tous les deux en sortiraient perdants. Sous cette double contrainte, le travail de figuration - ce que Goffman (1967 : 5-46) appelle face-work - est le mécanisme fondamental par lequel le Client et le Dealer tentent de rétablir l'équilibre sans cesse perturbé par l'incontournable confrontation.

Il est important en ce sens de comprendre que les règles de l'interaction entre le Dealer et le Client ne sont pas substantielles, mais plutôt cérémonielles. Selon Goffman (1967: 54) : 
[...] a ceremonial rule is one which guides conduct in matters felt to have secondary or even no significance in their own right, having their primary importance - officially anyway - as a conventionalized means of communication by which the individual expresses his character or conveys his appreciation of the other participants in the situation.

Ce qui compte, comme nous l'avons déjà vu, ce n'est pas le contenu de la transaction, mais l'expression de caractère qu' elle implique. Cela crée une situation où seul un des interactants peut être le gagnant, empêchant un accord pragmatique ou utilitaire conduisant à un résultat gagnant-gagnant du type supposé dans les modèles de choix rationnel qui mettent en scène l'homo ceconomicus. La confrontation dans le champ de coton ne dépend d'aucun échange particulier de marchandises qui permettrait au Client et au Dealer de se retrouver au point d'équilibre où leurs utilités marginales seraient optimisées. Elle repose plutôt sur la définition du rôle de chaque participant et, surtout, sur la force relative de cette définition par rapport à la définition de l'opposant.

Ainsi, l'interaction est constamment tendue par deux types d'actes de figuration : ceux qui visent à renforcer la propre image et ceux qui visent à renforcer l'image de l'autre pour maintenir ainsi le " consensus de travail "(Goffman, 1959 : 10). Il est évident que, dans un cadre purement agonistique, sans aucune forme de ritualisation, les premiers l'emporteraient toujours sur les seconds. Dans ce cas-là, le conflit serait vite résolu, en tant que l'un des adversaires s'imposerait sur l'autre en utilisant, ou en menaçant d'utiliser, la violence physique. Cependant, dans le champ de coton, un nonlieu hautement ritualisé, la dynamique résultant de ce travail de figuration contradictoire, qui découle de la double contrainte dans laquelle le Dealer et le Client sont pris, donne lieu à une interaction dialogique nécessairement alambiquée, caractérisée par de constants passages à un ordre métaphorique du message, avec des échanges à double ou triple sens, des actions stratégiques et des mouvements d'avance et de recul. Ainsi, la résolution se trouve sans cesse différée, produisant un discours qui est à la fois poétique et schizoïde, dans la mesure où il tourne « en spirale, en des distorsions sans fin, mais toujours systématiques " (Bateson, $1980: 19$ ).

Examinons d'abord les actions visant à maintenir le consensus, ou ce que Goffman (1967: 56-57) appelle la " déférence » :

By deference I shall refer to that component of activity which functions as a symbolic means by which appreciation is regularly conveyed to a recipient of this recipient, or of something of which this recipient is taken as a symbol, extension, or agent. These marks of devotion represent ways in which an actor celebrates and confirms his relation to a recipient. 
Dans les interactions quotidiennes, la déférence peut prendre la forme de salutations, de compliments ou d'excuses, entre autres comportements assez courants. Goffman (1967 : 57) appelle ces activités des "rituels interpersonnels » et distingue deux types principaux : les rituels d'évitement, destinés à préserver la distance interpersonnelle, ou ce que Simmel (1997 : 43) décrit comme la "sphère idéale ", et les rituels de présentation, destinés à renforcer la proximité ou la cohésion sociale.

Ces activités, essentielles pour maintenir le consensus de travail, on peut aussi les retrouver dans le non-lieu liminal du champ de coton. Lorsque le Dealer s'approche du Client, par exemple, il s'engage dans un clair rituel de présentation (Koltès, 1986 : 9-10) :

C'est pourquoi je m'approche de vous, malgré l'heure qui est celle où d'ordinaire l'homme et l'animal se jettent sauvagement l'un sur l'autre, je m'approche, moi, de vous, les mains ouvertes et les paumes tournées vers vous, avec l'humilité de celui qui propose face à celui qui achète, avec l'humilité de celui qui possède face à celui qui désire.

Bien entendu, cette attitude d'humilité initiale de la part du Dealer est un stratagème à double sens, qui vise à contraindre le Client à exprimer en premier lieu son besoin et, par conséquent, sa faiblesse. Mais dans sa forme extérieure, avec les mains offertes à l'autre en signe de bonne volonté, c'est aussi un acte de consensus cérémonial. Bref, c'est un rituel destiné à maintenir ouverte l'interaction malgré la brutalité implicite de la rencontre. Un peu plus tard, le Dealer dit au Client (Koltès, 1986 : 14) :

Je m'approche de vous comme le crépuscule approche cette première lumière, doucement, respectueusement, presque affectueusement, laissant tout en bas dans la rue l'animal et l'homme tirer sur leurs laisses et se montrer sauvagement les dents.

Le Client utilise aussi la déférence, mais pour souligner la distance cérémonielle qui le sépare du Dealer et, en même temps, car nous avons déjà vu que presque tous les actes ont plus d'un sens, pour renforcer son rôle dans le concours de caractères (Koltès, $1986: 32)$ :

C'est vous qui êtes le familier de ces lieux, et j'en suis l'étranger ; je suis celui qui a peur et qui a raison d'avoir peur ; je suis celui qui ne vous connaît pas, qui ne peut vous connaître, qui ne fait que supposer votre silhouette dans l'obscurité.

Ceci est un exemple de rituel d'évitement déployé au moment où la position de force du Dealer, après qu'il a obligé le Client à reconnaître la possibilité d'une transaction et attend qu'il nomme son objet, menace de briser le précaire équilibre rituel en introduisant ce que Goffman (1959: 137) appelle une " perturbation de la performance ». 
Malgré sa ritualisation extrême, la conversation entre le Dealer et le Client suit des modèles que nous pouvons également trouver dans les interactions sociales ordinaires. Selon Goffman (1967 : 76) :

[...] social intercourse involves a constant dialectic between presentational rituals and avoidance rituals. A peculiar tension must be maintained, for these opposing requirements of conduct must somehow be held apart from one another and yet realized together in the same interaction: the gestures which carry an actor to a recipient must also signify that things will not be carried too far.

Un exemple de cette dialectique est l'attitude d'humilité du Dealer évoquée cidessus. Le rituel de présentation s'accompagne dans ce cas d'un rituel d'évitement qui consiste à attribuer l'arrogance au Client : «C'est pourquoi j'emprunte provisoirement l'humilité et je vous prête l'arrogance, afin que l'on nous distingue l'un de l'autre à cette heure qui est inéluctablement la même pour vous et pour moi » (Koltès, 1986 : 12).

Nous ne devons pas oublier, cependant, qu'il ne s'agit pas ici d'une interaction ordinaire, mais d'un concours de caractères. La dynamique consensuelle des rituels de présentation et d'évitement se déploie à la surface d'une confrontation fatidique, portée par des actions visant à renforcer l'image de soi au détriment de l'image de l'autre. Ainsi, d'une part, nous avons le travail de figuration positive réalisé par chacun des acteurs au fur et à mesure qu'ils se présentent ou, selon les termes de Goffman (1967 : 77), communiquent leur « comportement ». En même temps, tous deux se livrent à la "profanation rituelle " (Goffman, 1967 : 88), c'est à dire, à une série d'actes visant à profaner l'image symbolique projetée par l'adversaire. C'est dans la dialectique entre comportement et profanation que l'on retrouve les éléments fondamentaux de ce concours.

Le comportement [demeanor], qui équivaut au travail de figuration sur la propre image ou à la présentation de soi, est une tentative de produire par des moyens symboliques une certaine identité individuelle ou ipséité. Cette identité n'existe pas en dehors de l'interaction sociale et doit être symboliquement réalisée pour être efficace, tout comme l'efficacité de la magie du shaman dépend exclusivement de son action symbolique (Lévi-Strauss, 1974 : 205-226). Pour illustrer cela, Goffman renvoie dans The Presentation of Self in Everyday Life au célèbre exemple de Sartre : quelqu'un est garçon de café parce qu'il se comporte comme un garçon de café, c'est-à-dire qu'il exécute une série d'actions symboliques avec lesquelles il projette l'image du garçon de café qu'il prétend être et que les autres acceptent de reconnaître comme telle (Sartre, 1976 : 95-96). Mais Goffman va encore plus loin. Inspiré par l'idée durkheimienne de la personnalité individuelle comme parcelle du mana collectif (Durkheim, 1912), ainsi que par la conceptualisation du rituel comme expression symbolique avec des fonctions 
sociales spécifiques chez Radcliffe-Brown (1952), Goffman conçoit ce travail de figuration comme une forme d'action rituelle. C'est un rituel parce qu'il est exécuté sur un objet sacré : l'image de soi ou le visage. "One's face, then, is a sacred thing, and the expressive order required to sustain it is therefore a ritual one " (Goffman, $1967: 19$ ).

Ainsi, lorsque le Client prétend appartenir à un autre monde, celui des lumières électriques et du commerce légal, il revendique le caractère sacré de son visage ; il se présente, non pas comme un sujet de désir, ce qui le livrerait aussitôt au Dealer, mais comme un sujet transcendantal, une revendication sur laquelle il assoit sa supériorité sur le Dealer (Koltès, 1986 : 18-19) :

Peut-être suis-je putain, mais si je le suis, mon bordel n'est pas de ce monde-ci; il s'étale, le mien, à la lumière légale et ferme ses portes le soir, timbré par la loi et éclairé par la lumière électrique, car même la lumière du soleil n'est pas fiable et a des complaisances. Qu'attendez-vous, vous, d'un homme qui ne fait pas un pas qui ne soit homologué et timbré et légal et inondé de lumière électrique dans ses moindres recoins?

La tentative du Dealer de le charger d'un désir coupable, de lui attribuer un certain besoin qui ne pourrait être satisfait que dans le monde souterrain et liminal, soulève la protestation la plus scandaleuse de la part du Client : "parce que, du seul poids de ce regard sur moi, la virginité qui est en moi se sent soudain violée " (Koltès, $1986: 19-20)$.

Le Dealer, quant à lui, assume d'emblée le rôle de celui qui habite le limen, quelqu'un qui a le pouvoir de satisfaire les désirs les plus secrets de ceux qui l'approchent et n'a qu'à attendre qu'ils lui passent la commande, mais n'a besoin de rien luimême. Et c'est justement sur ceci que repose sa supériorité sur le Client (Koltès, 1986 : 11) :

Voilà pourquoi je sais, mieux que l'acheteur inquiet qui garde encore un temps son mystère comme une petite vierge élevée pour être putain, que ce que vous me demanderez je l'ai déjà, et qu'il vous suffit, à vous, sans vous sentir blessé de l'apparente injustice qu'il y a à être le demandeur face à celui qui propose, de me le demander.

Et pourtant, malgré sa condition liminale, ou justement à cause d'elle, le visage du Dealer est aussi sacré que celui du Client (Koltès, 1986 : 16) :

Vous avez raison de penser [dit-il] que je ne descends de nulle part et que je n'ai nulle intention de monter, mais vous auriez tort de croire que j'en éprouve du regret. J'évite les ascenseurs comme un chien évite l'eau. Non pas qu'ils refusent de m'ouvrir leur porte ni que je répugne à m'y enfermer; mais les ascenseurs en mouvement me chatouillent et j'y perds ma dignité ; et, si 
j'aime être chatouillé, j'aime pouvoir ne plus l'être dès que ma dignité l'exige.

Il s'agit ici d'actes symboliques (et notamment, d'actes de langage) visant à réaliser le visage que chaque acteur veut imposer à l'autre. Ainsi, le Client prétend être un sujet transcendantal qui ne manque et n'a donc besoin de rien, tandis que le Dealer prétend être un sujet liminal qui possède déjà tout et donc n'a lui non plus besoin de rien. Dans cet effort pour prévaloir dans le concours de caractères, ces comportements sont aussi importants que les tentatives de chaque acteur pour abîmer le visage de l'autre à travers les profanations rituelles avec lesquelles ils s'attaquent à chaque tournant.

"Décidez-vous, montrez-vous : êtes-vous la brute qui écrase le pavé, ou êtesvous commerçant ? dans ce cas étalez votre marchandise d'abord, et l'on s'attardera à la regarder " (Koltès, 1986 : 27). Avec cette injonction, le Client essaie de déstabiliser le visage du Dealer, de l'exposer comme un voyou ordinaire qui cherche à l'emporter, non pas en montrant la supériorité de son caractère, mais par des moyens violents, par la force brute. Le Dealer est donc obligé de se défendre : «C'est parce que je veux être commerçant, et non brute, mais vrai commerçant, que je ne vous dis pas ce que je possède " (Koltès, 1986 : 27). En même temps, il contre-attaque et tente à son tour de faire tomber le visage du Client, l'exposant comme une bête dominée par le désir, afin de l'entraîner plus au fond dans le limen où il peut revendiquer sa supériorité : «Et parce que je vois le vôtre [désir] apparaître comme de la salive au coin de vos lèvres que vos lèvres ravalent, j'attendrai qu'il coule le long de votre menton ou que vous le crachiez avant de vous tendre un mouchoir " (Koltès, 1986 : 29). C'est alors que le Client se sent obligé de justifier une fois de plus son innocence, sa supériorité : "Car votre main s'est posée sur moi comme celle du bandit sur sa victime " (Koltès, 1986 : 33).

Nous pourrions continuer ainsi, en suivant les attaques et les parades, les contre-attaques et les ripostes, qui jusqu'au bout éperonnent l'action dramatique dans cette confrontation entre le Client et le Dealer. Avant de conclure, cependant, je n'examinerai qu'un des moments où le caractère rituel et symbolique apparaît avec plus de clarté. À la suite du dialogue, on apprend que le Dealer, lors de sa rencontre avec le Client, lui aurait offert son manteau (rituel de présentation). Mais le Client l'aurait laissé tomber au sol et aurait même craché dessus. Un tel acte a nécessairement de sérieuses implications rituelles, comme le fait remarquer le Dealer lui-même (Koltès, $1986: 58)$ :

Il n'est pas convenable pour un homme de laisser insulter son habit. Car si la vraie injustice de ce monde est celle du hasard de la naissance d'un homme, du hasard du lieu et de l'heure, la seule justice, c'est son vêtement. L'habit d'un homme c'est, mieux que lui-même, ce qu'il a de plus sacré. 
Dans ce passage, il apparaît plus explicite qu'ailleurs le fait que la confrontation se déploie dans une arène symbolique, où les profanations rituelles constituent des formes d'agression qui ne peuvent être laissées sans réponse. Le Client est donc pressé de rétablir l'équilibre rituel (Koltès, 1986 : 58) :

Eh bien je vous propose l'égalité. Une veste dans la poussière, je la paie d'une veste dans la poussière. Soyons égaux, à égalité d'orgueil, à égalité d'impuissance, également désarmés, souffrant également du froid et du chaud. Votre demi-nudité, votre moitié d'humiliation, je les paie de la moitié des miennes.

Le concours reste ainsi ouvert et il ne sera résolu que lorsque l'un des deux adversaires réussira à imposer son caractère à l'autre selon les normes non énoncées du rituel, "le sens des convenances" (Koltès, 1986: 35) que les deux acceptent "sans savoir pourquoi " (39). Car finalement, comme tous les deux le remarquent, la confrontation se résume à ceci, sachant qui des deux, si le Client ou le Dealer, pourra l'emporter sur l'autre. "Méfiez-vous du marchand ", avertit le Dealer, "son discours a l'apparence du respect et de la douceur, l'apparence de l'humilité, l'apparence de l'amour, l'apparence seulement" (Koltès, 1986 : 54). Ce à quoi le Client répond : "Méfiez-vous du client: il a l'air de chercher une chose alors qu'il en veut une autre, dont le vendeur ne se doute pas, et qu'il obtiendra finalement» (Koltès, 1986 : 59).

\section{Conclusion}

Si l'interaction entre le Client et le Dealer est tellement problématique et conséquente, donc fatale, c'est après tout parce que le champ de coton constitue l'archétype des non-lieux liminaux où se forment tous les systèmes sociaux. Selon Niklas Luhmann $(2010: 154)$ :

[...] les systèmes sociaux émergent du fait (et uniquement du fait) que les deux partenaires [ego et alter] font l'expérience de la double contingence et que l'indétermination d'une telle situation pour les deux partenaires dans n'importe quelle activité qui aurait lieu ensuite prend une signification pour la formation des structures.

Ce que l'interaction dans le champ de coton vient montrer, cependant, c'est que la double contingence n'agit pas toujours comme un facteur autocatalytique conduisant nécessairement à l'émergence du système social. De même, la rencontre de la face de l'autre n'est pas nécessairement, comme le veut Levinas (1984), un appel à la responsabilité éthique. En fait, comme l'explique Derrida (2006 : 30) dans son séminaire sur « l'animal », le regard de l'autre

[...] me donne à voir la limite abyssale de l'humain : l'inhumain ou l'anhumain, les fins de l'homme, à savoir le passage des frontières depuis lequel l'homme ose s'annoncer à lui-même, s'appelant ainsi du nom qu'il croit se donner. 
Dans ce cas-ci, ni le Client ni le Dealer ne peuvent renoncer au « concours de caractères » dans lequel ils se sont emmêlés. En dépit de leurs plaidoiries de plus en plus désespérées, ils ne parviennent pas à surmonter la double contrainte dans laquelle ils sont pris pour parvenir à un accord utilitaire quelconque et éviter une chute dans l'anomie, l'échec définitif du rituel qui exposerait leur « vie nue », qui dévoilerait finalement leur animalité (Agamben, 1992 et 2006). C'est en ce sens qu'ils se ressemblent, en fait, à beaucoup d'autres animaux. Mais non pas, comme l'humanisme a besoin de le croire, parce que la rupture du rituel leur enlèverait ce qui est censé les rendre proprement des êtres humains. Au contraire, c'est justement parce qu'ils sont subordonnés à l'« invariance des ordres rituels " (Rappaport, 1999: 44), à cette ritualisation incontournable des interactions qui régit au fond tous les rapports sociaux, qu'ils soient humains ou non (voir Watanabe \& Smuts, 2004), qu'ils ne réussissent pas à se mettre d'accord, à se compromettre. La dernière opportunité pour parvenir à une transaction, qui les aurait forcés tous les deux à accepter que le concours de caractères ne puisse pas avoir un vainqueur lorsqu'on s'est engagé dans la coopération, sombre dans l'abîme de la communication ratée (Koltès, $1986: 61$ ) :

LE DEALER : S'il vous plaît, dans le vacarme de la nuit, n'avez-vous rien dit que vous désiriez de moi, et que je n'aurais pas entendu?

LE CLIENT: Je n'ai rien dit ; je n'ai rien dit. Et vous, ne m'avez-vous rien, dans la nuit, dans l'obscurité si profonde qu'elle demande trop de temps pour qu'on s'y habitue, proposé, que je n'aie pas deviné ?

LE DEALER : Rien.

LE CLIENT : Alors, quelle arme ?

Le système social émergent s'effondre ainsi dans la méfiance mutuelle; la confrontation symbolique conduit à la violence ; le concours de caractères cède la place à un duel à mort. Comme le dit le Dealer, «il n'y a pas de règle; il n'y a que des moyens ; il n'y a que des armes " (Koltès, 1986 : 60). Dans le champ de coton, dans ce non-lieu liminal où se rencontrent deux inconnus, un acheteur et un vendeur, mais cela aurait pu être un homme et une femme, un blanc et un noir, un homosexuel et un maquereau, un immigrant et un citoyen, un chasseur et un éléphant, la résolution est toujours incertaine ; les combats peuvent être à mort, ne serait-ce que parce qu'il n'y a nulle part où se retirer, pas de règles et pas de lois, pas de droits qui peuvent être imposés et défendus. Dans ce limen sans identité, sans relations, et sans histoire, la vie nue des animaux, humains ou non humains, au fond peu importe, devient un rituel vide, la cérémonie quotidienne de la solitude. 


\section{RÉFÉRENCES BIBLIOGRAPHIQUES}

AgAmBEN, Giorgio (1997) : Homo sacer: Le pouvoir souverain et la vie nue. Traduction de Marilène Raiola. Paris, Éditions du Seuil.

Agamben, Giorgio (2006) : L'ouvert : De l'homme et de l'animal. Traduction de Joel Gayraud. Paris, Éditions Payot \& Rivages.

AUGÉ, Marc (1992) : Non-lieux : Introduction à une anthropologie de la surmodernité. Paris, Éditions du Seuil.

BATESON, Gregory (1980) : Vers une écologie de l'esprit. Traduction de Ferial Drosso et Laurencine Lot, avec le concours d'Eugène Simion et Christian Cler. Paris, Éditions du Seuil, t. 2.

CEFAÏ, Daniel \& Laurent PERREAU [dir.] (2012) : Erving Goffman et l'ordre de l'interaction. Paris, CURAPP-ESS/CEMS-IMM.

DERRIDA, Jacques (2006) : L'animal que donc je suis. Paris, Éditions Galilée.

DURKHEIM, Émile (1912) : Les formes élémentaires de la vie religieuse. Paris, Presses Universitaires de France.

GoffMAn, Erving (1959) : The Presentation of Self in Everyday Life. New York, Anchor Books.

GoffMan, Erving (1963) : Behavior in Public Places. New York, The Free Press.

GOFFMAN, Erving (1967) : Interaction Ritual : Essays on Face-to-face Behavior. New York, Pantheon Books.

KOLTÈS, Bernard-Marie (1986) : Dans la solitude des champs de coton. Paris, Les Éditions de Minuit.

LEVINAS, Emmanuel (1984) : Éthique et infini. Paris, Le Livre de Poche.

LÉVI-STRAUSS, Claude (1974) : Anthropologie structural. Paris, Librairie Plon.

LUHMANN, Niklas (2010) : Les systèmes sociaux. Esquisse d'une théorie générale. Traduction de Lukas K. Sosoe. Québec, Presses de l'Université Laval.

RADCLIFFE-BROWN, Alfred Reginald (1952) : Structure and Function in Primitive Society: Essays and Addresses. New York, The Free Press.

RAPPAPORT, Roy A. (1999) : Ritual and Religion in the Making of Humanity. Cambridge, Cambridge University Press.

RIBÓ, Ignasi (2020a) : «Posthuman political ecology : actors, networks, systems ». Conference Proceedings. The 6th International Conference on International Relations and Development (ICIRD) 2019, Mae Fah Luang University, Chiang Rai, Thaïlande. URL : https://www.icird2019.com/uploads/paper-5ee1b04712336-17\%20Full\%20paper\%20-\%20Ignasi_compressed.pdf

RIBÓ, Ignasi (2020b) : "Golden Triangle : a material-semiotic geography ». ISLE. Interdisciplinary Studies in Literature and Environment, isaa140. DOI: https://doi.org/10.1093/isle/isaa140.

SARTRE, Jean-Paul (1976) : L'être et le néant: Essai d'ontologie phénoménologique. Paris, Gallimard. 
SIMMEL, Georg (1997) : Simmel on Culture : Selected Writings. Édition de David Frisby et Mike Featherstone. New York, SAGE Publications.

TURNER, Victor (1969) : The Ritual Process : Structure and Anti-structure. Chicago, Aldine Publishing Company.

WATANABE, John M. \& Barbara B. SMUTS (2004) : «Cooperation, commitment, and communication in the evolution of human sociality ", in Robert W. Sussman \& Audrey R. Chapman (éd.), The Origins and Nature of Sociality. New York, Aldine de Gruyter, 288-309. 